\title{
ATITUDES DE ENFERMEIROS FRENTE AO ALCOOLISMO: REVISÃO DA LITERATURA
}

Divane Vargas ${ }^{1}$, Janaina Soares ${ }^{2}$

\begin{abstract}
RESUMO: Trata-se de uma revisão sistemática que objetivou investigar e analisar as publicações relacionadas às atitudes de enfermeiros frente ao álcool, ao alcoolismo e ao alcoolista, publicadas entre 1959 e 2009 . Realizou-se a busca sistemática nas bases de dados PUBMED, MEDLINE, LILACS e SciELO, utilizando-se os descritores attitudes; nurse; alcohol e alcoholism, resultando numa amostra constituída por 38 artigos. Os resultados apontaram que houve aumento na produção sobre o tema na década de 1990, e a análise qualitativa dos dados permitiu evidenciar que existe maior aceitação do álcool e que seus dependentes nas últimas décadas, porém, ainda há uma parcela de enfermeiros com atitudes predominantemente negativas ou moralistas. Concluiu-se que a produção sobre as atitudes de enfermeiros frente às questões relacionadas ao álcool indexadas nas bases de dados disponíveis online ainda é pequena se comparada a outros assuntos relacionados à temática, o que pode indicar pouco interesse dos pesquisadores sobre o assunto.
\end{abstract}

PALAVRAS-CHAVE: Atitudes; Enfermeiros; Alcoolismo; Pesquisa em Enfermagem.

\section{ATTITUDES OF NURSES TO ALCOHOLISM: A LITERATURE SEARCH}

ABSTRACT: A systematic revision which aimed to investigate and analyse publications related to the attitudes of nurses to alcohol, alcoholism and alcoholics, published between 1959 and 2009. A systematic search was carried out using the databases PUBMED, MEDLINE, LILACS and SciELO, using the search parameters attitudes, nurse, alcohol and alcoholism, resulting in a sample composed of 38 articles. The results indicated that there has been an increase in the number of articles since 1990, and qualitative analysis of the articles allowed it to be argued that in recent decades there has been greater acceptance of alcohol and its dependents, although there are still many nurses whose attitudes are predominantly negative or moralistic. It was concluded that the production of articles on the attitude of nurses to matters related to alcohol indexed in the databases available on-line is still small when compared to other subjects related to the theme, which may indicate a lack of interest among researchers in the question.

KEYWORDS: Attitudes; Nurses; Alcoholism; Nursing research.

\section{ACTITUDES DE ENFERMEROS DELANTE DEL ALCOHOLISMO: REVISIÓN DE LITERATURA}

RESUMEN: Esta es una revisión sistemática que tuvo el objetivo de investigar y analizar las publicaciones referentes a las actitudes de enfermeros delante del alcohol, del alcoholismo y del alcoholista, publicadas entre 1959 y 2009. Se realizó la búsqueda sistemática en las bases de datos PUBMED, MEDLINE, LILACS y SciELO, utilizándose los descriptores attitudes; nurse; alcohol y alcoholism, resultando en una muestra constituida por 38 artículos. Los resultados apuntaron que huvo aumento en la producción sobre el tema en la década de 1990, y el análisis cualitativo de los datos permitió evidenciar que existe mayor aceptación del alcohol y que sus dependientes en las últimas décadas, pero, todavía hay una parcela de enfermeros con actitudes predominantemente negativas o moralistas. Se concluyó que la producción sobre las actitudes de enfermeros delante de cuestiones acerca del alcohol indexadas en as bases de datos disponibles on-line todavía es pequeãa en comparación a otros asuntos relacionados a la temática, lo que puede indicar poco interés de los investigadores sobre el asunto.

PALABRAS CLAVE: Actitudes; Enfermeros; Alcoholismo; Investigación en Enfermería

\footnotetext{
${ }^{1}$ Enfermeiro. Doutor em Enfermagem Psiquiátrica. Professor do Departamento de Enfermagem Materno-Infantil e Psiquiátrica e do Programa de Pós-Graduação da Escola de Enfermagem da Universidade de São Paulo.

${ }^{2}$ Enfermeira. Mestre em Enfermagem.
}

\author{
Autor correspondente: \\ Divane de Vargas \\ Universidade de São Paulo \\ Av. Dr. Enéas de Carvalho Aguiar, 419 - 05403-000 - São Paulo-SP -Brasil \\ E-mail: vargas@usp.br
}

Recebido: 01/06/10

Aprovado: $15 / 10 / 10$

Cogitare Enferm. 2011 Abr/Jun; 16(2):340-7 


\section{INTRODUÇÃO}

Os problemas relacionados ao uso do álcool e alcoolismo vêm aumentando significativamente; estima-se que, mundialmente, quase dois bilhões de pessoas consumam bebidas alcoólicas e 76,3 milhões apresentem algum tipo de desordem por causa do uso do álcool ${ }^{(1)}$.

No Brasil, de 2001 e 2005 houve um aumento de $11,2 \%$ para $12,3 \%$ de dependentes do álcool entre a população com idade entre 12 e 65 anos $^{(2)}$. Em decorrência disso, cada vez mais o enfermeiro, independente do local onde atue, está sujeito a ter contato com pessoas com problemas relacionados ao álcool. É comum, por exemplo, encontrar pacientes com problemas diretamente relacionados com o alcoolismo em unidades clínicas e cirúrgicas de hospitais gerais ${ }^{(3)}$, bem como em unidades de atenção primária à saúde ${ }^{(4)}$. Nas unidades de emergência são frequentes os atendimentos à vítimas de acidentes e de violência associados ao abuso do álcool ${ }^{(5)}$. O enfermeiro, além de prestar assistência ao usuário de álccol, desenvolve atividades com sua família e com crianças e jovens expostos ao álcool.

Quando um alcoolista chega para atendimento, independentemente do serviço que procure, é provável que seu primeiro contato se dê com o enfermeiro e demais profissionais da equipe de enfermagem. Sendo assim as atitudes que estes profissionais apresentem frente ao paciente poderão afetar diretamente o curso do tratamento subsequente. No entanto, apesar das atitudes e percepções de trabalhadores da saúde exercerem grandes implicações na resposta do paciente, tanto no tratamento de desordens orgânicas como psíquicas $^{(6)}$, existem poucos estudos investigando as atitudes desses profissionais de saúde e, especificamente, as atitudes do enfermeiro frente ao alcoolismo e ao alcoolista.

A constatação leva a pressupor que, apesar da relevância desse tema para a Enfermagem, os enfermeiros pouco investigam sobre a questão ou não divulgam os estudos. Assim, esse fato por si só justifica a necessidade de estudos que se ocupem de levantar e caracterizar a produção da Enfermagem sobre a temática. Por considerar a escassez de estudos que avaliem as atitudes de enfermeiros na temática em questão, e a reconhecida importância de se identificar o conhecimento produzido pela área nas últimas décadas, este estudo tem como objetivo investigar e analisar as publicações relacionadas às atitudes de enfermeiros frente ao álcool, ao alcoolismo e ao alcoolista nas últimas cinco décadas.

\section{METODOLOGIA}

Esta é uma pesquisa exploratória de cunho bibliográfico. Para a coleta dos dados, realizou-se uma busca sistemática nas bases de dados PUBMED - National Library of Medicine, MEDLINE - Medical Literature Analysis and Retrieval System Online, LILACS - Literatura Latino-Americana e do Caribe em Ciências da Saúde e SciELO - Scientific Eletronic Library Online. Nessa busca, foram utilizados os seguintes descritores: 1. attitudes; 2. nurse, 3. alcohol and alcoholism, posteriormente, foram usadas também as fontes adicionais em busca assistemática em periódicos. Na amostra, foram incluídos estudos que abordam as atitudes de enfermeiros frente ao álcool, ao alcoolismo e ao alcoolista, escritos em língua portuguesa, inglesa ou espanhola e publicados entre 1959 e 2009 . A escolha desse período deveu-se ao fato de que, no final da década de 1950, começaram a ser publicados os primeiros estudos relacionados à questão. Na busca sistemática foram identificadas 60 publicações, e na busca assistemática nas fontes adicionais foram encontradas três.

Depois de concluída a etapa de coleta de dados, 38 artigos preencheram os critérios de inclusão na amostra. De posse do material, os artigos foram distribuídos conforme a década em que foram publicados, país de origem e população do estudo. Feita esta classificação, procedeu-se a segunda fase de análise, representada pela análise qualitativa das publicações, o que exigiu uma abordagem qualitativa na qual se utilizou a análise de conteúdo ${ }^{(7)}$ para a categorização do material. Esse tratamento caracterizou-se pela leitura exaustiva das publicações, procurando determinar como as atitudes dos enfermeiros frente ao álcool, ao alcoolismo e ao alcoolista têm sido abordadas nas publicações dos últimos 50 anos.

A análise qualitativa das publicações permitiu a categorização do material com base nos dados evidentes nas publicações (elementos componentes) ${ }^{(7)}$ que foram agrupados por semelhanças, originando quatro categorias para análise: I. Examinando as atitudes dos enfermeiros frente ao paciente alcoolista; II. As atitudes após treinamentos e capacitações; III. Estudos experimentais sobre as atitudes; IV. Comparando as atitudes entre grupos de enfermeiros.

\section{RESULTADOS}

Ao analisar as produções sobre as atitudes dos enfermeiros frente ao álcool, ao alcoolismo e ao alcoo- 
lista, observou-se que houve um aumento significativo da produção científica nas duas últimas décadas, e que o número de publicações sobre o tema duplicou, quando se compara esse número de publicações entre as décadas de 1990 a 2009 (Figura 1).

No que se refere à origem do conhecimento publicado, os resultados permitem observar que a maioria dos estudos foi realizada nos Estados Unidos da América $(16 ; 42,1 \%)$, seguida do Brasil $(13 ; 34,2 \%)$, Inglaterra (4;10,5\%), e Escócia (2;5,3\%). Além disso, foram encontrados também trabalhos do Japão, Austrália e Canadá, que contribuíram com um artigo cada, correspondendo a 7,9\% da amostra analisada. A análise qualitativa das publicações foi realizadas a partir das quatro categorias temáticas:

\section{I - Examinando as atitudes dos enfermeiros frente ao paciente alcoolista}

Esta categoria agrupa 28 publicações referentes a estudos que se ocuparam em mensurar as atitudes dos enfermeiros na temática em estudo, nos diversos cenários de atuação desses profissionais. Os dados dessas pesquisas foram em sua maioria coletados pela aplicação de escalas, questionários e estudos de caso e permitem examinar como tem se mostrado as atitudes desses profissionais ao longo das últimas cinco décadas.

Nos Estados Unidos da América e em alguns países da Europa, os estudos ${ }^{(8-19)}$ tiveram, em sua maioria, o objetivo de identificar atitudes do enfermeiro nos diversos cenários de atuação desse profissional, dentre eles, hospitais gerais ${ }^{(11-13,15-17)}$, atenção primária ${ }^{(19)}$, clínicas especializadas ${ }^{(10)}$, ambulatórios, departamentos de emergências ${ }^{(20)}$, entre outros.

A análise do material possibilitou observar que, no final da década de 1950 e início da década de 1960, nos Estados Unidos da América, surgiram os primeiros estudos sobre atitudes dos enfermeiros frente à problemática do álcool. As evidências desses estudos foram de que os enfermeiros acreditavam que o tratamento do alcoolista era desesperançoso ${ }^{(8)}$, que os enfermeiros que tinham contato direto com usuários de álcool apresentavam sentimentos ambivalentes ou negativos sobre o cuidar dessa clientela ${ }^{(9)}$, e que os enfermeiros de maior nível educacional e menor faixa etária apresentavam atitudes mais positivas frente ao álcool e alcoolismo ${ }^{(10)}$. Na década de 1970, não foi encontrado estudo sobre a temática, nas bases pesquisadas.

Os estudos da década de 1980 mostraram que os enfermeiros consideravam o paciente alcoolista como mais responsável por seus problemas de saúde do que os pacientes tidos como não alcoolistas ${ }^{(11)}$. Surgem também estudos ${ }^{(12)}$ relacionados ao conhecimento do profissional enfermeiro frente ao alcoolismo, evidenciando que esses relatavam não ter conhecimento suficiente para detectar o alcoolismo entre os pacientes e sentiam-se frustrados diante das recaídas de pacientes alcoolistas ${ }^{(13)}$. Observa-se também que os enfermeiros de clínicas médicas e cirúrgicas apresentavam maior aceitação do conceito do alcoolismo como doença médica e orgânica do que as alocadas em unidades psiquiátricas $^{(14)}$.

Na década de 1990, observa-se um avanço nas pesquisas relacionadas às atitudes dos enfermeiros frente às questões do álcool ${ }^{(16-18)}$, e a problemática começa a despertar maior interesse dos pesquisadores, inclusive brasileiros ${ }^{(21)}$. De acordo com a análise dessas pesquisas ${ }^{(16)}$, pela primeira vez observa-se que fatores emocionais são apontados pelos enfermeiros como causa importante do controle de uso do álcool.

Figura 1 - Distribuição das publicações (n 38) sobre as atitudes de enfermeiros frente ao álcool, ao alcoolismo e ao alcoolista entre 1950 a 2009. São Paulo, 2010.

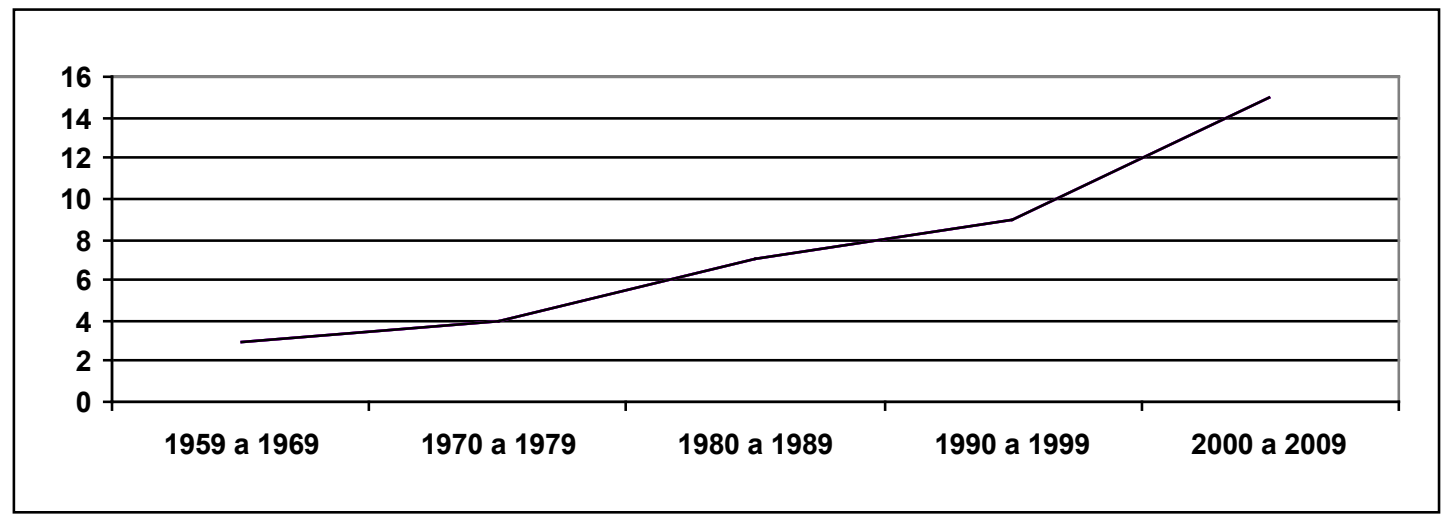


Nota-se, também nessa década, que os enfermeiros, embora concebam os alcoolistas como pessoas doentes, continuam preferindo não trabalhar com os mesmos ${ }^{(17)}$ e constata-se o despreparo desses profissionais em relação à abordagem e ao tratamento dos problemas relacionados ao alcoolismo ${ }^{(18)}$. No final da década de 1990 foi publicado o primeiro estudo brasileiro sobre a questão, avaliando os instrumentos para a mensuração de atitudes de enfermeiros frente ao alcoolismo ${ }^{(21)}$.

Nos anos 2000, o interesse pelo temática atitudes frente ao álcool, ao alcoolismo e ao alcoolista mantevese entre os pesquisadores de todo o mundo, inclusive do Brasil. Tais estudos evidenciaram concepções negativas dos enfermeiros com pacientes alcoolistas ${ }^{(22)}$, a falta de preparo de enfermeiros da atenção primária com usuários de álcool ${ }^{(23)}$; mesmo sendo aqueles que mantêm contato constante com pacientes com problemas relacionados ao álcool e alcoolismo, os enfermeiros não se sentem preparados para identificação e manejo desses problemas.

No Brasil, pesquisas sobre a temática ${ }^{(24-25)}$ também evidenciaram que os enfermeiros de atenção básica eram favoráveis ao tratamento para o alcoolismo, sendo o paciente percebido como doente. Porém, apresentaram atitudes negativas frente à satisfação em trabalhar com essa clientela, se comparados aos enfermeiros que trabalhavam em unidades hospitalares, pois estes acreditavam que os alcoolistas eram pessoas fisicamente doentes, devendo submeter-se a tratamento médico ${ }^{(26)}$; esses enfermeiros também revelaram sentir-se embaraçados ao trabalhar com indivíduos que apresentam problema com o álcool ${ }^{(26)}$. Em relação ao conhecimento, os estudos brasileiros ${ }^{(27-28)}$ mostraram que os enfermeiros apresentavam dificuldade de reconhecer os problemas relacionados ao uso do álcool e identificar sua relação com outras patologias clínicas.

De modo geral, a análise dos estudos permitiu observar que os pacientes alcoolistas são vistos como pacientes mais responsáveis pela sua doença do que outros, não alcoolistas. Na Atenção Primária à saúde, os profissionais enfermeiros também demonstraram despreparo e falta de habilidade frente ao tratamento do alcoolista, considerando que quando esses profissionais são treinados ou capacitados, se sentem mais dispostos e aptos a cuidar desse paciente, além de diminuir suas atitudes negativas frente ao tratamento.

Ao analisar as produções que se ocuparam de mensurar as atitudes de enfermeiros frente ao álcool, ao alcoolismo e ao alcoolista, observa-se que elas começaram a aparecer somente na década de 1980, período em que se apontam as primeiras concepções desses profissionais de que fatores emocionais exercem influência no desenvolvimento do alcoolismo. Já o final da década de 1990 e início dos anos 2000 são marcados pelo aumento expressivo da produção sobre a temática, quando comparada a períodos anteriores.

\section{II - As atitudes após treinamentos e capacitações}

A segunda categoria é composta por elementos de quatro publicações relacionadas à mensuração das atitudes dos enfermeiros frente às questões relacionadas ao álcool, ao alcoolismo e ao alcoolista geralmente, abordando os resultados provenientes de pesquisas realizadas com esses profissionais antes e após algum tipo de capacitação ou treinamento para atuar na área.

Pesquisa que investigou as atitudes de enfermeiros que foram submetidos a um programa de treinamento em álcool e alcoolismo ${ }^{(29)}$, referindo-se ao tratamento e à motivação do alcoolista para se recuperar, encontrou que, após o treinamento, a maioria dos enfermeiros mostrou-se otimista com relação ao tratamento do alcoolismo, julgando ainda que o alcoolista precisa ser motivado para que o tratamento obtenha sucesso. No entanto, observou-se que a maioria dos enfermeiros continuava demonstrando visões moralistas a respeito do alcoolismo, mesmo após o treinamento ${ }^{(29)}$. $\mathrm{Na}$ avaliação dos resultados de um wokshop para conscientização de enfermeiros de saúde pública sobre os problemas de saúde decorrentes do uso de drogas, os autores aplicaram escalas de mensuração de atitudes e conhecimentos, antes e três meses após o treinamento. Observou-se que, após o treinamento, houve diminuição das atitudes permissivas em relação ao uso de drogas, concomitante a um aumento da confiança de enfermeiros em relação à eficácia do tratamento ${ }^{(30)}$.

Ao avaliar um programa de três anos, elaborado para melhorar as atitudes de enfermeiros frente a usuários de substâncias psicoativas e aumentar suas habilidades clínicas para o enfrentamento do problema, e desenvolvido por um grupo de enfermeiros de um hospital universitário, os autores ${ }^{(32)}$ constataram significativas mudanças na comparação entre o pré e pós-treinamento. As enfermeiras pós-treinamento apresentavam atitudes mais positivas frente ao paciente e estavam mais otimistas em relação ao tratamento ${ }^{(31)}$. Avaliando as atitudes de enfermeiras suecas que receberam treinamento e acompanhamento de especialistas para detecção e tratamento de problemas relacionados ao álcool durante 60 dias, os autores observaram que houve significativa mudança nas mesmas, com o predomínio de atitudes 
mais positivas após o acompanhamento ${ }^{(32)}$.

A partir de 2000, houve um aumento importante de estudos sobre treinamentos e capacitações referentes à questão do conhecimento dos profissionais frente ao alcoolismo. Constatou-se que a quantidade de conhecimentos recebidos pelos mesmos influencia positivamente em suas atitudes e percepções, após os treinamentos realizados. No que se refere à associação entre a aquisição de conhecimento e a mudança de atitudes, houve unanimidade em afirmar que existe relação entre os conhecimentos e as atitudes, e há necessidade de aprimorar os conhecimentos desses profissionais para que apresentem atitudes mais positivas frente à problemática do alcoolismo.

\section{III - Estudos experimentais sobre as atitudes}

Esta categoria é encontrada em três estudos que objetivaram a mensuração das atitudes de enfermeiros elegendo, em todos eles, um grupo experimental e um de controle, visando, dentre outros, avaliar o impacto do estereótipo do alcoolista nas atitudes dos profissionais. Os estudos puderam evidenciar que os efeitos de estereótipos negativos nas atitudes de enfermeiros exercem forte influência em suas atitudes frente ao paciente alcoolista ${ }^{(33)}$. Quando o paciente é rotulado como alcoolista, o profissional tem uma visão condenatória para o mesmo ${ }^{(34)}$; quando o paciente alcoolista é comparado a pacientes com outras patologias, $\mathrm{o}$ alcoolista tende a ser considerado pelos enfermeiros como mais responsável pelas suas ações do que outros pacientes ${ }^{(35)}$.

Ao analisar esses estudos experimentais sobre as atitudes dos enfermeiros, observa-se que o estereótipo negativo do paciente alcoolista e do alcoolismo exerce forte influência nas atitudes dos profissionais, contribuindo para que estas se tornem mais negativas. Evidencia-se o predomínio de concepções morais e condenatórias por parte de enfermeiros, que tende a culpar o alcoolista pela sua doença.

\section{IV - Comparando as atitudes entre grupos de en- fermeiros}

Esta categoria é oriunda de três artigos que procuraram estabelecer comparação das atitudes entre grupos de enfermeiros; a maioria ocupou-se de comparar as atitudes de enfermeiros que trabalhavam ou tinham contato com pacientes alcoolistas e os que não possíam tal experiência.

Na comparação de atitudes entre dois grupos de enfermeiros observou-se que os enfermeiros da uni- dade de internação eram mais favoráveis a abordagens humanistas e terapêuticas de tratamento do que os da unidade de emergência ${ }^{(36)}$. Já comparando as atitudes e conhecimentos entre enfermeiros de unidades gerais de internação e os enfermeiros de unidades especializadas em alcoolismo, ficou evidente que os enfermeiros de unidades gerais mostraram-se mais tolerantes ao ato de beber, mas tinham atitudes mais moralistas frente ao alcoolismo ${ }^{(37)}$. Entre os enfermeiros que utilizam os serviços de interconsulta em psiquiatria, para o atendimento de alcoolistas, e os enfermeiros que não empregavam essa estratégia, evidenciaram-se diferenças estatísticas nas atitudes dos dois grupos ${ }^{(38)}$.

Apesar do número reduzido de estudos que se ocuparam em comparar grupos de enfermeiros com relação às atitudes frente ao álcool, ao alcoolismo e ao alcoolista, o resultado de sua análise sugere que os enfermeiros que mantêm maior contato com pacientes alcoolistas ou intoxicados tenderam a ter atitudes mais negativas frente ao alcoolista; os enfermeiros de instituições especializadas no tratamento mostraram atitudes mais positivas.

\section{DISCUSSÃO}

O levantamento da produção científica relacionada às atitudes dos enfermeiros frente ao álcool, ao alcoolismo e ao alcoolista nas últimas cinco décadas evidenciou que o tema começou a despertar maior interesse dos pesquisadores a partir da década de 1990. Isso se evidencia pelo volumoso e diverso corpo de pesquisa que vem sendo acumulado nas últimas duas décadas, ao redor do mundo, sobre a temática.

Apesar de estudado nos Estados Unidos da América e em alguns países da Europa já há cinco décadas, aproximadamente ${ }^{(8-19)}$, no Brasil, o tema despertou interesse dos pesquisadores somente no final da década de 1990 e início do século XXI. O fato parece justificar o reduzido número de publicações sobre a temática na literatura nacional ${ }^{(22-28)}$, quando comparada com a literatura de outros países ${ }^{(8-19)}$. De acordo com especialistas ${ }^{(39)}$, o aumento considerável na produção de Enfermagem sobre a temática álcool e outras drogas a partir da década de 1990, pode ser um reflexo do aumento da produção científica da Enfermagem em geral. Nesse espaço de tempo houve também um aumento nos programas de pós-graduação, cujos órgãos de avaliação passaram a exigir mais publicações científicas e redução no tempo de obtenção das titulações (mestres e doutores). 
No que se refere aos temas que têm sido abordados, destacam-se os estudos com o objetivo de examinar as atitudes dos enfermeiros frente ao álcool, ao alcoolismo e ao alcoolista ${ }^{(8-28)}$. Os estudos visando a identificar a influência de capacitações e treinamentos nas atitudes de enfermeiros também têm chamado a atenção dos pesquisadores sobre o tema ${ }^{(29-32)}$; esses estudos ocupamse de mensurar as atitudes de enfermeiros antes e após treinamentos. A análise dos resultados permite evidenciar que, geralmente, depois desses treinamentos, há um aumento significativo do conhecimento sobre o tema. No entanto, essa mesma influência não é observada na mudança das atitudes dos enfermeiros, que tendem a permanecer as mesmas ou discreta melhora quando comparadas com situações pré-teste ${ }^{(29)}$.

Outra abordagem das atitudes dos enfermeiros frente à temática tem sido feita por meio de estudos experimentais, onde o pesquisador, geralmente, verifica a influência de uma variável (informação sobre o uso do álcool ou alcoolismo por parte do paciente) nas atitudes dos enfermeiros. Esses trabalhos têm indicado que os estudos experimentais com enfermeiros ${ }^{(33-35)}$ apontam que um paciente identificado como alcoolista é percebido mais negativamente em relação a uma série de atributos pessoais do que um paciente idêntico que não é tido como tal.

Com relação aos locais de pesquisa de atitudes frente ao álcool, ao alcoolismo e ao alcoolista, predominam os estudos realizados com enfermeiros de unidades hospitalares $^{(11-13,15-17)}$ e alguns com enfermeiros do trabalho e de serviços de atenção primária à saúde ${ }^{(19,23)}$.

Conforme constatado nos resultados dos estudos realizados no exterior e no Brasil, as atitudes de enfermeiros frente ao abuso de substâncias parecem ser mais positivas na atualidade do que há três décadas, sendo possível se evidenciar esta tendência sobretudo nos estudos publicados a partir da década de 1980 . Mas, esses mesmos estudos ${ }^{(11-15)}$ revelam que uma significativa proporção de enfermeiros ainda continua apresentando atitudes moralistas e condenatórias frente aos dependentes de álcool.

Nos estudos comparativos ${ }^{(36-38)}$ é possível verificar que os enfermeiros que cuidam diretamente dessa população tendem a ser menos permissivos frente ao uso pessoal e social do álcool, além de apresentarem atitudes menos condenatórias frente aos dependentes ${ }^{(36)}$. Esses enfermeiros tendem ainda a possuir maior conhecimento sobre a questão e mostram-se mais otimistas com relação ao prognóstico do paciente alcoolista ${ }^{(36-37)}$.

No que se refere à questão do conhecimento dos enfermeiros frente ao alcoolismo constata-se que a quantidade de informações influencia positivamente em suas atitudes e percepções frente ao alcoolista. Este dado pode ser constatado quando se avalia estudo em que os enfermeiros que se consideravam capazes de reconhecer os problemas decorrentes do beber excessivo sentiam-se mais confortáveis na interação com esses pacientes ${ }^{(9)}$. Segundo os resultados, parece que programas centrados apenas na aquisição de conhecimento com o intuito de mudar as atitudes dos enfermeiros, com respeito aos dependentes de álcool, têm resultado ineficaz causando pouco impacto nas atitudes. Além disso, a análise de estudo evidencia também que, apesar de terem ocorrido mudanças na visão que se tinha do alcoolista nos últimos anos, comprovadamente mais positivas, esses pacientes continuam sendo apontados entre os últimos tipos de pacientes com os quais os profissionais enfermeiros preferem trabalhar ${ }^{(27)}$.

\section{CONCLUSÃO}

Com relação aos resultados da pesquisa, o panorama geral estabelecido permitiu observar que a produção sobre a temática atitudes de enfermeiros frente ao álcool, ao alcoolismo e ao alcoolista cadastrada nas bases de dados disponíveis online ainda é pequena. Esse levantamento evidenciou que, até o final da década de 1990, não existia nenhum tipo de produção no Brasil, fato que atualmente se encontra em mudança, uma vez que quase todos os trabalhos publicados, entre $1999 \mathrm{e}$ 2009 (56,2\%), são oriundos de nosso país.

A revisão da literatura publicada nas últimas cinco décadas permitiu evidenciar que existe maior aceitação do uso do álcool e de seus dependentes pelos enfermeiros nos últimos anos. No entanto, uma parcela significativa desses profissionais continua a considerar os usuários dessas substâncias como imorais, com caráter falho e com baixa probabilidade de recuperação, preferindo não trabalhar com eles.

\section{REFERÊNCIAS}

1. World Health Organization (WHO). Collaborative study group on alcohol and injuries. WHO, Final report. Geneva: WHO; 2004.

2. Carlini EA, Galduróz JCF, Noto AR, Nappo SA. II Levantamento domiciliar sobre uso de drogas no Brasil - 2005. Centro Brasileiro de Informações Sobre Drogas Psicotrópicas, Departamento de Psicobiologia da Escola Paulista de Medicina e SENAD - Secretaria Nacional 
Antidrogas, Presidência da República, Gabinete de Segurança Nacional. Brasília; 2006.

3. Noto AR, Moura YG, Nappo SA, Galduróz JCF, Carlini EA . Internações por transtornos mentais e de comportamento decorrentes de substâncias psicoativas: um estudo epidemiológico nacional do período de 1988 a 1999. J Bras Psiquiatr. 2002;51(2):113-21.

4. Vargas D, Oliveira MAF, Araújo EC. Prevalência de dependência alcoólica em serviços de atenção primária à saúde de Bebedouro, São Paulo, Brasil. Cad Saúde Pública. 2009;25(8):1711-20.

5. Segatto ML, Pinsky L, Laranjeira R, Rezende FF, Vilela TR. Triagem e intervenção breve em pacientes alcoolizados atendidos na emergência: perspectivas e desafios. Cad Saúde Pública [Internet]. 2007;23(8) [acesso em 30 jun 2010]. Disponível: http://migre. $\mathrm{me} / 51 \mathrm{M} 2 \mathrm{j}$

6. Mogar RE. Staff attitudes towards the alcoholic patient. Arch Gen Psychiatry. 1969;21:449-54.

7. Bardin L. Análise do conteúdo. $5^{\mathrm{a}}$ ed. rev. atual. Lisboa: Edições 70; 2009.

8. Blane HT, Hill MJ. Public health nurses speak up about alcoholism. Nurs Outlook. 1964.

9. Johnson MW. Nurses speak out on alcoholism. Nurs Forum. 1965;4(4):16-22.

10. Heinemann E, Rhodes RJ. How nurses view the tuberculosis alcoholic patient. Nurs Res. 1967;16(4):3615.

11. Biener L. Perceptions of patients by emergency room staff: substance abusers versus non-substance abusers. J Health Soc Behav. 1983;24(3):264-75.

12. Reismam BL, Shrader RW. Effect of nurses' attitudes toward alcoholism on their referral rate for treatment. Occup Health Nurs. 1984;32(5):273-5.

13. Bartek JK, Lindeman M, Newton M, Fitzgerald AP, Hawks JH. Nurse-identified problems in the management of alcoholic patients. J Stud Alcohol. 1988;49(1):62-70.

14. Potamianos G, Winter D, Duffy SW, Gorman DM, Peters TJ. The perception of problem drinkers by general hospital staff, general practitioners and alcoholic patients. Alcohol. 1985;2(4):563-6.
15. Rowland N, Maynard AK. Alcohol education for patients: some nurses need persuading. Nurse Educ Today. 1989;9(2):100-4.

16. Allen K. Attitudes of registered nurses toward alcoholic patients in a general hospital population. Int J Addict. 1993;28(9):923-30.

17. Grief CL, Elliott R. Emergency nurses' moral evaluation of patients. J Emerg Nurs. 1994;20(4):275-9.

18. Cooper D. Problem drinking. Nurs Times. 1994;90(14):36-9.

19. Lock CA, Kaner E, Lamont S, Bond S. A qualitative study of nurses' attitudes and practices regarding brief alcohol intervention in primary health care. J Adv Nurs. 2002;39(4):333-42.

20. Chung JYM, Chan JTS, Yeung RSD, Wan RCH, Ho ST. Nurses' attitude toward alcoholic patients in accident and emergency department in Hong Kong. J Emerg Med. 2003;10(2):104-12.

21. Pillon CS. Atitudes dos enfermeiros com relação ao alcoolismo [dissertação]. São Paulo(SP): Universidade Federal de São Paulo; 1998.

22. Vargas D, Labate RC, Costa Junior ML. Alcoolistas tratar ou punir: disposição de enfermeiros de Hospital Geral. Rev Enferm UERJ. 2003;11(2):188-92.

23. Vargas D, Luis MAV. Álcool, alcoolismo e alcoolista: concepções e atitudes de enfermeiros de unidades básicas distritais de saúde. Rev Latino-Am Enfermagem. 2008;16(n. esp):543-50.

24. Vargas D, Labate RC. Trabalhar com pacientes alcoolistas: satisfação de enfermeiros de hospital geral. Rev Gaúch Enferm. 2005;26(2):252-60.

25. Vargas D, Labate RC. Atitudes de enfermeiros de hospital geral frente ao uso do álcool e alcoolismo. Rev Bras Enferm. 2006;59(1):47-51.

26. Vargas D, Luis MA. Construção e validação de uma escala de atitudes frente ao álcool, ao alcoolismo e ao alcoolista. Rev Latino-Am Enfermagem. 2008;16(5):895-902.

27. Lucca DM, Vargas D, Vargas D. As concepções de enfermeiros de hospital geral frente as questões relacionadas ao álcool e ao alcoolismo. Enferm Bras. 2006;5(5):260-6. 
28. Pillon CS, Luís MAV, Laranjeira R. Atitudes dos enfermeiros relacionadas ao alcoolismo. Nursing. 2006;96(9):811-6.

29. Rosebaun PD. Public health nurses in the treatment of alcohol abusers. Can J Public Health. 1977;68(6):503-8.

30. Hagemaster J, Handley S, Plumlee A, Sullivan E, Stanley S. Developing educational programmes for nurses that meet today's addiction challenges. Nurse Educ Today. 1993;13(6):421-5.

31. Gerace LM, Hughes TL, Spunt J. Improving nurses' responses toward substance-misusing patients: a clinical evaluation project. Arch Psychiatr Nurs. 1995;9(5):286-94.

32. Bendtsen P, Akerlind I. Changes in attitudes and practices in primary health care with regard to early intervention for problem drinkers. Alcohol. 1999;34(5):795-800.

33. Wallston KA, Wallston BS, DeVellis BM. Effect of a negative stereotype on nurses' attitudes toward an alcoholic patient. J Stud Alcohol. 1976;37(5):659-65.

34. Cornish RD, Miller MV. Attitudes of registered nurses toward the alcoholic. J Psychiatr Ment Health Serv. 1976;14(12):19-22.

35. Krueger J. Perceptions of sexual assault victim responsibility by emergency nurses when there was chemical use by victim. J Emerg Nurs. 1995;21:174-5.

36. Berger-Gross V, Lisman S. Attitudes of paraprofessionals toward alcoholism; setting effects. J Stud Alcohol. 1979;40(5):514-7.

37. Hisatomi N, Ito K, Nagashima Y, Fujiwara K, Yasuda K, Ashizawa T, et al. Nurses' knowledge of alcohol-related problems and their treatment: a comparison of nurses in general hospital and alcoholism treatment units. Nihon Arukoru Yakubutsu Igakkai Zasshi. 1998;33(6):671-82.

38. Happell B, Taylor C. Negative attitudes towards clients with drug and alcohol related problems: finding the elusive solution. Aust N Z J Ment Health Nurs. 2001;10(2):87-96.

39. Luis MAV, Lunetta ACF. Álcool e outras drogas: levantamento preliminar sobre a pesquisa produzida no Brasil pela enfermagem. Rev Latino-Am Enfermagem. 2005;3(esp):1219-30. 